\title{
FAQS: Fitting Automatic Quasar Spectra
}

\section{M. Śniegowska*}

Warsaw University Observatory, Al. Ujazdowskie 4, 00-478 Warsaw, Poland

Center for Theoretical Physics, Polish Academy of Sciences, Al. Lotników 32/46, 02-668

Warsaw, Poland E-mail: marzena.sniegowska@student.uw.edu.pl

\section{K. Hryniewicz}

Nicolaus Copernicus Astronomical Center, Polish Academy of Sciences, Bartycka 18, 00-716

Warsaw, Poland

\section{B. Czerny}

Center for Theoretical Physics, Polish Academy of Sciences, Al. Lotników 32/46, 02-668

Warsaw, Poland

\section{S. Panda}

Center for Theoretical Physics, Polish Academy of Sciences, Al. Lotników 32/46, 02-668

Warsaw, Poland

Nicolaus Copernicus Astronomical Center, Polish Academy of Sciences, Bartycka 18, 00-716

Warsaw, Poland

\begin{abstract}
We report the study of 2955 quasars, brighter than $m_{i}=18^{\text {mag }}$ and $m_{r}=18^{\text {mag }}$ at $z<0.7$, from the Sloan Digital Sky Survey. ${ }^{\dagger}$

We write a program to automate the fitting of AGN optical spectra. We fit the spectra taking into account the starlight contamination, reddening of the starlight and consider several Fe II templates.

We describe our fitting tool Fitting Automatic Quasar Spectra (FAQS). In the future work, our results will be compared to the Shen \& Ho (2014) diagram, and will be used to investigate the properties of the strong Fe II emitters, with the aim to identify the key physical mechanism leading to the efficient Fe II production.
\end{abstract}

Revisiting narrow-line Seyfert 1 galaxies and their place in the Universe - NLS1 Padova 9-13 April 2018

Padova Botanical Garden, Italy

\footnotetext{
* Speaker.

${ }^{\dagger}$ https://www.sdss.org/
} 


\section{Introduction}

Active galactic nuclei (AGN) are astrophysical sources powered by an accreting central super massive black hole, whose observed properties depend on inclination and brightness, according to the Unified AGN Model (Urry \& Padovani 1995). The principal components analysis (PCA) for 87 AGN was done by Boroson \& Green (1992), while Shen \& Ho (2014) analyzed the spectral properties for almost 20000 objects (taken from Shen et al. 2011 (S11)). To look for spectral properties of AGN and to fit their spectra (or spectral energy distribution) we need large samples and good software for spectral analysis.

The motivation to start writing automatized analysis procedure was caused by the fact that the spectral analysis IRAF packages, such as SPECFIT (Kriss 1994), does not suits well for the purpose of automatic fitting of thousands of sources. At the moment of making decision we found no publicly available software which would fill our needs. Additionally catalogs of spectral properties like S11 do not account for all spectral components (e.g., starlight). Recently independently developed software packages for automatic fitting of AGN spectra emerged, like QSFit (Calderone et al. 2017). However QSFit fits different models than we would like to apply. In this article we briefly describe our approach and the initial sample that we have chosen.

\section{Selection of the sample}

We select 2955 active galaxies from the $\mathrm{S} 11$ catalog, which satisfy criteria: $m_{i} \leq 18 \mathrm{mag}$, $m_{r} \leq 18 \mathrm{mag}$ and $z \leq 0.9$. Criterion for apparent magnitude ensures that data for these objects have good quality, whereas criterion for redshift ensures the presence of $\mathrm{H} \beta$ line in the optical spectrum. The selected sample is shown in the $\mathrm{R}_{F e}=\mathrm{EW}(\mathrm{FeII}) / \mathrm{EW}(\mathrm{H} \beta)$ vs. FWHM diagram (Fig. 1). Our sample simply moved towards the main cluster of points.

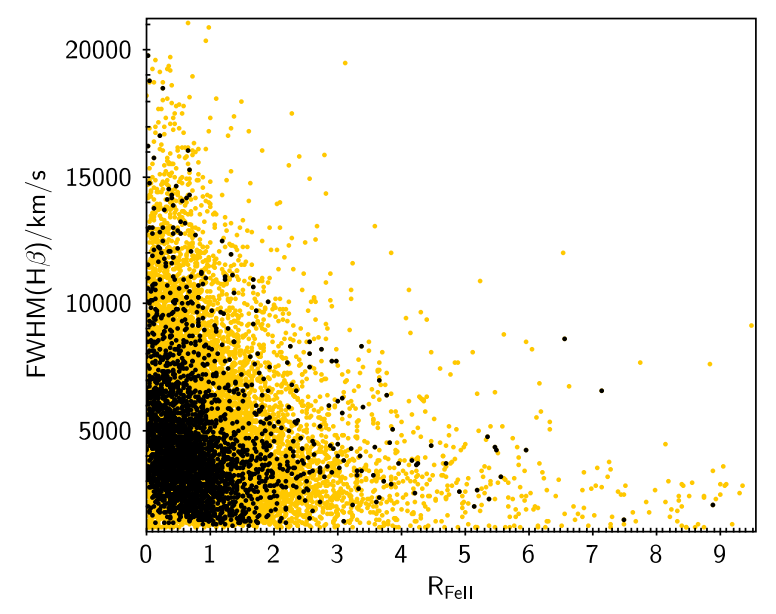

Figure 1: The diagram shows quasars main sequence. AGN with high quality spectra (black points) with comparison to the whole S11 catalog (orange points). 

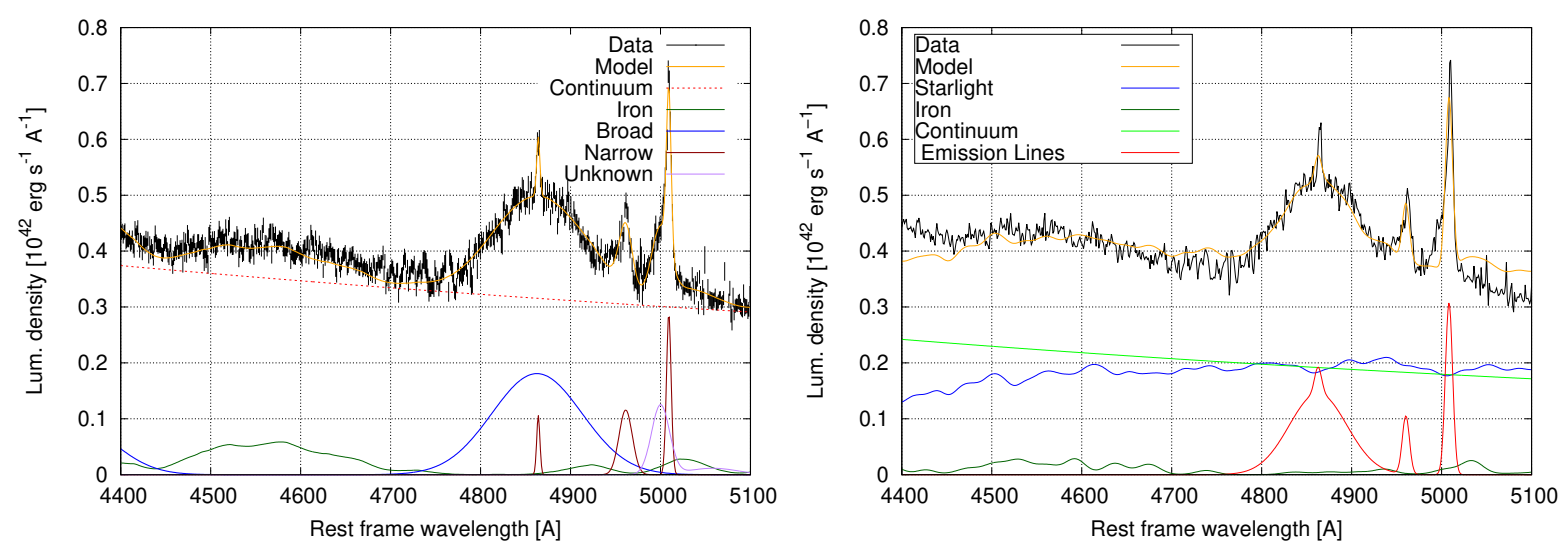

Figure 2: The expanded region of the $\mathrm{H} \beta$ line (magenta line) of the J000110.97-105247.4 fit, using QSFit (left panel) and using our routine (right panel). Our fit uses extinction correction based on $\mathrm{NED}^{a}$, and the data binning with resolution $1 \AA$. This causes the difference in the observed spectrum compared to the QSOfit output shown in the left panel.

${ }^{a}$ http://ned.ipac.caltech.edu/

\section{Fitting procedure}

The fitting tool we used is "Fitting Automatic Quasar Spectra (FAQS), which works as follows. Initially spectral windows covering emission line are masked. To model the starlight contribution we use the subsample of templates from Bruzual \& Charlot (2003). To approximate the thermal accretion disk emission we assume a power-law model. After first five iterations we fit various $\mathrm{Fe}$ II templates, using the Bruhweiler \& Verner (2008) templates to get the best $\chi^{2}$, after which the remaining 5 iterations are computed. We model in detail the wavelength region $4400-5100 \AA$, with lines $\mathrm{H} \gamma, \mathrm{H} \beta$, and the [OIII] doublet. Each object from the sample is modeled in 10 iterations.

\section{Comparison of methods in QSFit software and our program}

Methods used in our program and QSFit software have differences, which can affect the final results. The most significant of them are:

- In QSFit one elliptical galaxy template is used (Polletta et al. 2007), while in our program we use several templates representing combinations of various stellar populations (Bruzual \& Charlot (2003)).

- QSFit uses only one iron template (Véron-Cetty et al. 2004), while our program uses various iron templates (e.g. Bruhweiler \& Verner (2008)).

- In the last iteration in QSFit all parameters are free, in our program five parameters are free in each step.

We focus on efficiency, thus core procedures are written in Fortran while visualization and analytics capabilities are provided through Python. However it is still work in progress. QSFit is written in 
IDL as in the moment of writing. We compare fits of the same object using our routine with the QSFit model, both of them are presented in Fig. 2. The most visible differences are in iron templates and in asymmetric part of OIII 5007 $\AA$ line, which in QSFit case is modelled as the undefined line and in our routine left as unfitted part.

\section{Discussion}

The decomposition of the spectra is difficult. This is seen already in the S11 and QSFit catalog. We improved the analysis by taking into account several FeII templates and starlight contamination, but the objects are still not well fitted (like Fig. 2 (right panel)) and the work has to be improved. The work which is shown here is still in progress and in this paper, we just show how the procedure works on the one example, and compare it with QSFit fits. Methods used in our routine and QSFit have differences, which probably affect the final results.

\section{Acknowledgements}

This conference has been organized with the support of the Department of Physics and Astronomy "Galileo Galilei", the University of Padova, the National Institute of Astrophysics INAF, the Padova Planetarium, and the RadioNet consortium. RadioNet has received funding from the European Union's Horizon 2020 research and innovation programme under grant agreement No. 730562.

The project was partially supported by Polish grant No. 2015/17/B/ST9/03436/. The Fe II theoretical templates described in Bruhweiler \& Verner (2008) were downloaded from the web page http://iacs.cua.edu/personnel/personal-verner-feii.cfm with the permission of the authors. We thank Riccardo Nanni for his technical comments for this manuscript. We are also deeply grateful to the anonymous referee for multiple comments.

\section{References}

[1] Bruhweiler, F. \& Verner, E., 2008, ApJ, 675, 83

[2] Bruzual G. \& Charlot S., 2003, MNRAS, 344, 1000

[3] Boroson, T. A. \& Green, R. F., 1992, ApJS, 80, 109

[4] Calderone, G., Nicastro, L., Ghisellini, G., et al. 2017, MNRAS, 472, 4051

[5] Kriss, G. 1994, Astronomical Data Analysis Software and Systems III, 61, 437

[6] Polletta, M., Tajer, M., Maraschi, L., et al. 2007, ApJ, 663, 81

[7] Shen, Y. et al., 2011, ApJS, 194, 45

[8] Shen, Y. \& Ho, L. C., 2014, Nature, 513, 210

[9] Śniegowska, M., Czerny, B., You, B., et al. 2018, A\&A, 613, A38

[10] Urry, C. M. \& Padovani, P. 1995, PASP, 107, 803

[11] Véron-Cetty, M.-P., Joly, M., \& Véron, P. 2004, A\&A, 417, 515 